\title{
Arachnoid hyperplasia in optic nerve glioma: confusion with orbital meningioma
}

\author{
ROBERT J. COOLING AND JOHN E. WRIGHT \\ From Moorfields Eye Hospital, City Road, London
}

SUMMARY A case is reported of an optic nerve glioma with a marked degree of arachnoid hyperplasia which was initially diagnosed as an optic nerve meningioma. Hyperplasia of the arachnoid was also the underlying cause for expansion of the optic canal. The relationship between arachnoid hyperplasia in optic nerve glioma and meningioma of the optic nerve sheath in childhood is discussed.

Arachnoid hyperplasia is a meningeal response which is often seen in association with gliomas of the anterior visual pathways. This case report concerns an example of exuberant arachnoid hyperplasia affecting the intracranial and intracanalicular portions of the optic nerve excised through a transcranial approach. The specimen comprised a normal optic nerve surrounded by meningothelial cells and psammoma bodies and was interpreted as a primary optic nerve meningioma. An optic nerve glioma was subsequently removed through a lateral orbital approach, so that the true diagnosis was established. This paper discusses the implications of these findings and offers an explanation for the high incidence of primary optic nerve meningiomas in children reported by Karp et al. (1974), figures at variance with those previously published from this hospital.

\section{Case report}

An 8-year-old girl first presented in April 1974 to Moorfields Eye Hospital. There was a 4-month history of left sided painless proptosis and a left convergent squint. She had previously been healthy. The visual acuity in the left eye was reduced to $6 / 36$ with a left convergent squint of $40^{\circ}$. There were $3 \mathrm{~mm}$ of axial nonpulsatile proptosis, upward movement of the eye was restricted, and there was a marked left afferent pupillary defect. The left optic disc appeared hypoplastic, with swelling of the nerve head visible in the upper nasal quadrant. The right visual acuity was $6 / 6$, with intact central and peripheral visual fields. There were no other

Correspondence to R. J. Cooling, FRCS, Moorfields Eye Hospital, City Road, London EC1V 2PD. abnormal neurological findings and specifically no stigmata of neurofibromatosis.

Plain radiographs of the optic foramina showed enlargement of the left canal with a regular and intact margin. The right optic foramen was normal. Widening of the left optic canal throughout its length was confirmed on axial polytomography. B-scan ultrasonography showed an acoustically homogeneous mass arising from the left optic nerve. The pattern visual evoked response showed a normal response on the right side but diminished amplitude and increased latency on the left.

An exploratory biopsy of the left optic nerve was undertaken in April 1974, revealing a bluish distension of the optic nerve sheath. Histopathological examination showed an area of meningothelial cells, fibrous tissue, and a few well-differentiated glial cells. There was no evidence of optic nerve glioma in the sections examined.

In October 1977 the visual acuity in the left eye was further reduced to 6/60. Proptosis of the left eye had increased to $10 \mathrm{~mm}$ with $8 \mathrm{~mm}$ of inferior displacement. The left optic disc showed generalised pallor, with diffuse nerve fibre loss in the retina visible on red-free ophthalmoscopic examination. Computerised axial tomography showed an illdefined expansion of the left optic nerve (Fig. 1). The density of the lesion was considerably enhanced after the administration of contrast medium (Conray 420). The intracranial scan with particular reference to the parasellar region was normal. An air encephalogram showed a normal intracranial optic nerve on the left side.

A left frontal craniotomy was performed in November 1977. The chiasm and intracranial optic nerves appeared entirely normal. Emerging from the left optic canal was a greyish mass which encircled 


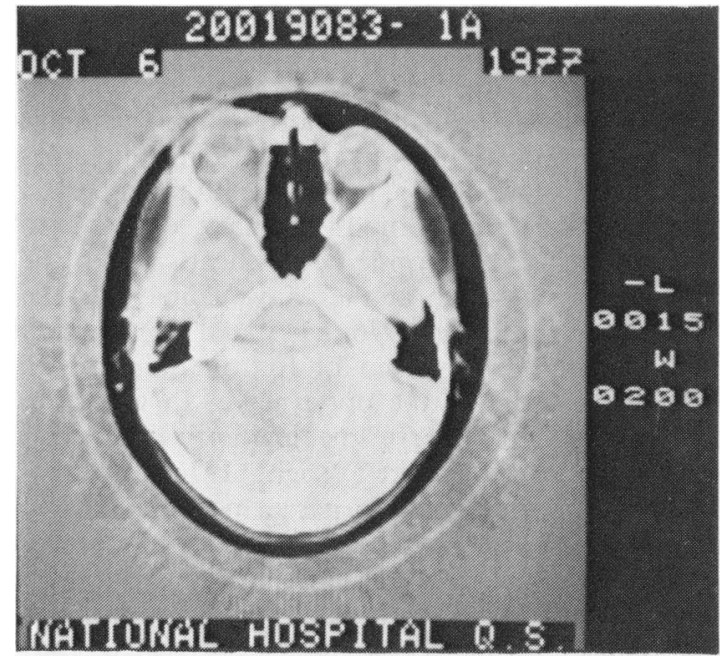

Fig. 1 Computerised tomographic scan shows large irregular mass in left retrobulbar space

the optic nerve. Transection of the left optic nerve was performed at its junction with the chiasm and reflected forwards through a deroofed optic canal. The nerve was divided $1 \mathrm{~mm}$ anterior to the common tendon within the orbit.

Histopathological examination showed a normal optic nerve with an extensive collar of meningothelial cells and an intermingling of psammoma bodies. No evidence of glial hyperplasia or astrocytic glioma was seen throughout the intracanalicular or intracranial portions of the optic nerve. The diagnosis was a primary optic nerve meningioma.

In February 1978 a left orbitotomy was carried out via a lateral canthotomy. A large bluish globular mass continuous with the optic nerve was excised, and the optic nerve was divided flush with the globe. Fig. 2 shows the eccentric disposition of the tumour in relation to the optic nerve. Histological examination showed a typical grade I (Reese, 1976) astrocytic glioma of the optic nerve with extensive meningothelial cell proliferation in a whorled pattern and numerous psammoma bodies.

The patient was last examined 12 months after orbitotomy. There was no evidence of orbital recurrence or intracranial tumour, and the cosmetic appearance was good.

\section{Discussion}

Proliferation of arachnoid cells was observed by Verhoeff (1922) in several cases of optic nerve glioma. Distension of the perineural space by meningothelial cells was seen proximal and distal to the underlying glioma. Proliferation of conti- guous tissue elements has been noted in the connective tissue trabeculae of the optic nerve (Kiel, 1923), in the capillaries within the optic nerve glioma, and the supporting tissues of the arachnoid. Davis (1940) emphasised the development of arachnoid hyperplasia, which appeared to follow extension of the glioma beyond the pial sheath of the optic nerve. Arachnoid hyperplasia is seen as an orderly arrangement of meningothelial or cap cells with or without psammoma bodies. Extension of the glioma or of the arachnoid hyperplasia rarely occurs beyond the dura mater.

Examples of prolific meningeal hyperplasia have formed the basis of several case reports. Walter and McKenney (1964) reported arachnoid hyperplasia with cyst formation as the cause for progressive proptosis following excision of an optic nerve glioma. Sanders et al. (1965) described a case of extensive arachnoid hyperplasia within the orbit in an intracranial optic nerve glioma in which invasion of the orbit by glioma was not demonstrable.

The possibility of diagnostic confusion between arachnoid hyperplasia accompanying a glioma, and orbital meningioma has previously been raised (Hogan and Zimmerman, 1962; Walsh and Hoyt, 1969). The case reported here illustrates the manner in which meningioma may be diagnosed when hyperplastic arachnoid remote from the glioma is examined. The histological appearance complete with the presence of psammoma bodies and the normal underlying optic nerve made the histological diagnosis inevitable, while the assessment was made

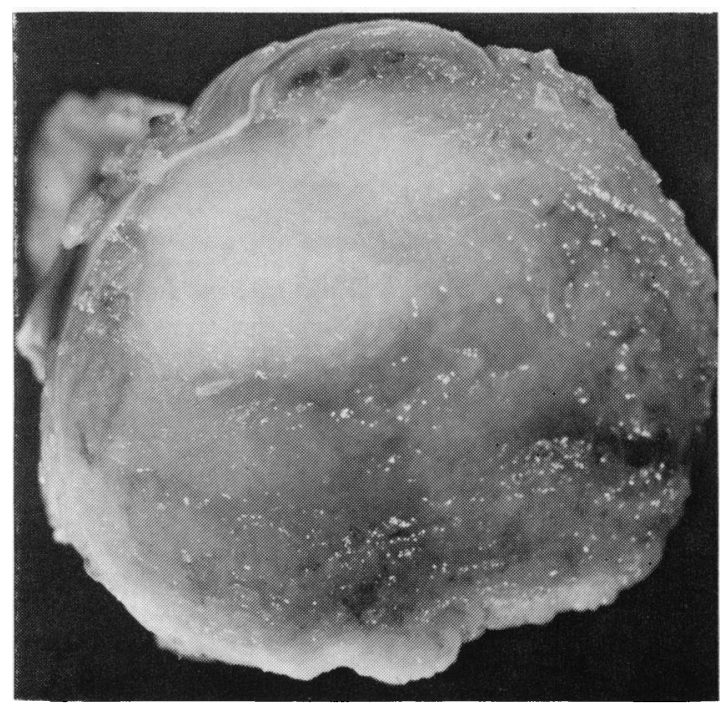

Fig. 2 Cut surface of orbital tumour. $\times 3$ 


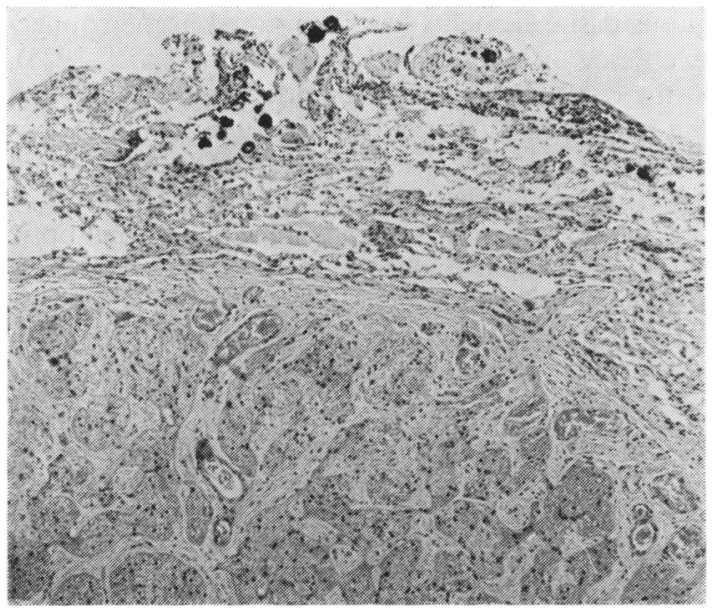

Fig. 3 Photomicrograph of transverse section of intracranial portion of optic nerve. There is meningeal hyperplasia in which psammoma bodies are seen. Haematoxylin and eosin, $\times 44$

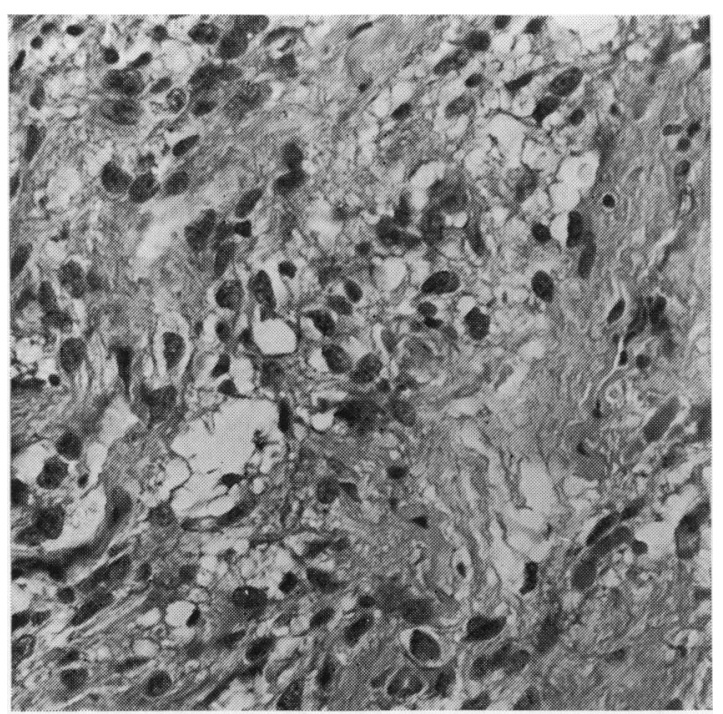

Fig. 4 Photomicrograph of glioma showing neoplastic proliferation of glial cells together with a fibrillary component. Haematoxylin and eosin, $\times 270$

without the full clinical history and findings. This case also demonstrates that expansion of the optic canal can be caused by arachnoid hyperplasia rather than extension of the glioma beyond the confines of the orbit. Spencer (1972) recorded a similar cause for optic canal enlargement in an orbital glioma.

The origin of orbital meningiomas from the arachnoid sheath of the optic nerve was at one time questioned (Verhoeff, 1932). Several series of orbital meningiomas have now been reported which establish the optic nerve sheath as the commonest origin of primary intraorbital meningiomas. Craig and Gogela (1949) reported 9 meningiomas arising from the optic nerve sheath. Henderson (1973) considered 11 of 14 primary tumours to have arisen from the optic nerve sheath within the orbit in patients seen at the Mayo Clinic over an 18-year period. Reese (1976) has stated that primary orbital meningiomas account for one-third of all primary tumours of the optic nerve. In the series of primary optic nerve meningiomas previously reported from this centre (Wright, 1976) a total of 15 cases of primary optic nerve meningioma was reported. It was emphasised that meningiomas arising from the optic nerve within the orbit were seen in adults with a female preponderance. Since that report a further 5 cases have been treated. Only 1 child aged 11 years has been encountered out of the 20 patients examined. This is in agreement with several previously reported series (Craig and Gogela, 1949; Henderson, 1973; Reese, 1976).

The relative incidence of this tumour in the first 2 decades has been brought into question by the report by Karp et al. (1974) from the Armed Forces Institute of Pathology. This was a multicentric series between the years 1925 and 1968 . Twenty-five patients were considered to have a meningioma arising from the optic nerve. Ten patients $(40 \%)$ were younger than 20 years and 6 $(24 \%)$ were less than 10 years of age. They concluded that primary intraorbital meningiomas are less rare in children than has generally been assumed and that their data supported the suggestion by Walsh (1975) that primary orbital meningiomas in childhood are more aggressive than those seen in adult life. The case material for this study was a clinicopathological audit extended over a 43-year period.

From the data published in this paper it is apparent that great reliance was placed on the histopathological interpretation of excised tissue. This could well cause errors, for the clinical details were provided by clinicians remote from the laboratory and in many cases well before the advent of sophisticated methods of investigation such as computerised tomographic scanning and ultrasound. The case reported in our communication has shown that the differentiation between gliomas and optic nerve meningiomas cannot rest on histopathology in isolation. We would therefore suggest that the results published by Karp et al. (1974) may be incorrect and that some of their cases in the younger age group were in reality gliomas. Certainly the disparity between their figures and those published 
from this hospital (Wright, 1976) and by other authors (Craig and Gogela, 1949; Henderson, 1973; Reese, 1976) can be explained on this basis.

The authors thank Dr J. Harry and the Department of Pathology, Institute of Ophthalmology, for their assistance with the histopathology; the Department of Radiology, National Hospital Queen Square, for supplying the computerised tomographic scan, and Mrs S. Cole for typing the manuscript.

\section{References}

Craig, W. McK., and Gogela, L. J. (1949). Intraorbital meningiomas; a clinicopathological study. American Journal of Ophthalmology, 32, 1663-1680.

Davis, F. A. (1940). Primary tumours of the optic nerve (a phenomenon of von Recklinghausen's disease). Archives of Ophthalmology, 23, 735-821, 957-1022.

Henderson, J. W. (1973). Orbital Tumours, pp. 527-554. Saunders: Philadelphia.

Hogan, M. J., and Zimmerman, L. E. (1962). Ophthalmic Pathology, an Atlas and Textbook, 2nd edn. Saunders: Philadelphia.

Karp, L. A., Zimmerman, L. E., Borit, A., and Spencer, W. H. (1974). Primary intraorbital meningiomas. Archives of Ophthalmology, 91, 24-28.

Kiel, E. (1923). Zur histologie der Opticustumoren. Albrecht von Graefes Archiv für Ophthalmologie, 112, 64-79.
Reese, A. B. (1976). Tumours of the Eye, 3rd edn., pp. 148153. Harper and Row: New York.

Sanders, G. S., Allen, R. A., and Straatsma, B. R. (1965). Arachnoidal proliferation of optic nerve simulating extension of intracranial glioma. Archives of Ophthalmology, 74, 349-352.

Spencer, W. H. (1972). Primary neoplasms of the optic nerve and its sheath: clinical features and current cocepts of pathogenetic mechanisms. Transactions of the American Ophthalmological Society, 70, 490-528.

Verhoeff, F. H. (1922). Primary intraneural tumours (gliomas) of the optic nerve. Archives of Ophthalmology, 51, 120-140, 239-254.

Verhoeff, F. H. (1932). Tumours of the optic nerve. In Penfield, W. (ed.), Cytology and Cellular Pathology of the Nervous System, Vol. 3, pp. 1029-1039. Paul B. Hoeder: New York.

Walsh, F. B. (1975). Meningiomas primary within the orbit and optic canal. In Glaser, J. S., and Smith, J. L. (eds.), Neuro-ophthalmology, Vol. 8, pp. 166-190. C. V. Mosby: St Louis.

Walsh, F. B., and Hoyt, W. F. (1969). Clinical Neuroophthalmology, 3rd edn., Vol. 3, p. 2076. Williams and Wilkins: Baltimore.

Walter, J. R., and McKenney, M. J. (1964). Collateral hyperplasia and cyst formation of orbital leptomeninx. American Journal of Ophthalmology, 57, 1037-1042.

Wright, J. E. (1976). Primary optic nerve meningiomas: clinical presentation and management. Transactions of the American Academy of Ophthalmology and Otolaryngology, 83, 617-625. 